\title{
An anti-n-methyl-d-aspartate receptor antibody syndrome-like presentation and negative result of testing for autoantibodies in a Saudi boy
}

\begin{abstract}
Autoimmune encephalitis is a diverse group of neuropsychiatric disorders recognized recently. It gained attention because of its favorable response to immunotherapy. This report describes a boy whose distinct and remarkable stepwise clinical picture of non-specific prodrome, psychiatric, neurological and autonomic symptoms suggested the possibility of anti-N-methyl-D-aspartate receptor antibody encephalitis. He had lymphocytic pleocytosis in his cerebrospinal fluid, abnormal electroencephalogram results with normal magnetic resonance imaging, and negative anti-NMDA receptor antibodies both in the serum and cerebrospinal fluid. He responded to combined immunotherapy of pulse steroid and intravenous immunoglobulin, with steady improvement over 4 weeks to almost complete remission for 4 years, with no reported relapses. Tumor surveillance initially and later on did not reveal any hidden tumors. This case highlights the importance of early recognition of this disorder and the commencement of immunotherapy even in the absence of antibodies as early therapy improves the long-term neurologic outcome in those patients.
\end{abstract}

Keywords: Anti-NMDA encephalitis, autoimmune encephalitis, immunotherapy
Volume 9 Issue I - 2019

\author{
Amal Y Kentab, MD \\ Department of Pediatrics, King Khalid University Hospital, King \\ Saud University, Riyadh, Saudi Arabia
}

\begin{abstract}
Correspondence: Dr.Amal Y. Kentab, Associate Professor \& Consultant Pediatric Neurologist, Department of Pediatrics (39), King Khalid University Hospital, College of Medicine, King Saud University, P.O. Box 2925, Riyadh I I46I, Saudi Arabia, Emailamkentab@hotmail.com, akentab@ksu.edu.sa
\end{abstract}

Received: January 07, 2019 | Published: February 06, 2019

\section{Introduction}

Autoimmune encephalitis is a diverse group of neuropsychiatric disorders recognized recently. It gained attention because of its favorable response to immunotherapy. Based on epidemiological studies, anti-N-methyl-D-aspartate (NMDAR) encephalitis is the most common cause of autoimmune encephalitis, ranking immediately after acute demyelinating encephalomyelitis (ADEM). ${ }^{1}$

It is associated with anti-NMDA ( $\operatorname{IgG})$ receptor antibodies, which bind to extracellular epitopes in the NR1 and NR2 heteromers of the NMDA receptor. Its pathogenesis remains unknown; however, evidence of antibody-mediated encephalitis is supported by the disappearance of antibodies during remission and the favorable response to immunotherapy. It was established by Josep Dalmau and colleagues in $2007 .^{2}$ It has been identified mostly in females with first-onset psychiatric symptoms, in association with paraneoplastic syndrome secondry to ovarian teratomas, but it can also be found in the absence of any evident tumor. ${ }^{3}$ It is becoming increasingly recognized in children and affects both sexes, and 30-50\% of affected children exhibit associated neoplasms. ${ }^{3-5}$

Its clinical presentation is characterized by four main stages: nonspecific prodrome initially, followed by psychiatric symptoms ${ }^{6}$ with rapid progression to the last neurological stage, before the recovery stage started in Table 1. It is confirmed by detection of NMDA receptor antibodies in serum and/or cerebrospinal fluid. Patients benefit from combined immunotherapy and early tumor resection in paraneoplastic form. ${ }^{7}$ Recovery is usually slow and can take years, and the outcome is usually favorable, but neuropsychological sequelae and relapses have been reported. ${ }^{4,8}$

This report describes a 6-year-old male patient with a typical clinical presentation suggestive of anti-N-methyl-D-aspartate receptor encephalitis. He did not manifest the antibody, although he responded to combined immunotherapy.

\section{Case report}

A previously healthy 6-year-old boy presented with two days' history of sudden onset of unprovoked generalized tonic-clonic seizures, occurring 3 times a day and lasting less than 10 minutes. At home he was noted to have extreme fatigability, anorexia, excessive sleepiness, slowness in thinking and inappropriate affect. His previous development was normal, and his immunizations were up to date. Five days before presentation, he had viral-like illness with fever and gastroenteritis-like picture, with vomiting and diarrhea. His history was free of any psychiatric disorders like anxiety or social phobia. No family history of any neurologic or movement disorder was reported.

On examination, he was not oriented with regard to time, but was oriented in regard to place and person. He was withdrawn and lethargic but intermittently agitated, responded reluctantly to questions, manifested slowness of thinking and constructional apraxia, and often appeared confused. He had normal cranial nerves, no nystagmus, but had difficulty in performing finger-to-nose-to-finger maneuvers because of his apraxia. Other cerebellar, sensory, motor, and gait examinations produced normal results.

He was admitted to intensive care unit to control his frequent Left-sided partial seizures with secondry generalization. Intravenous midazolam and multiple antiepileptic drugs, including phenytoin, phenobarbitone, topiramate and carbamezapine were tried. His condition worsened, he became completely withdrawn, and he would not read, write or follow commands. He manifested confusion, impaired memory, bouts of agitation, bizarre behavior, visual hallucination, and movement disorders in the form of orofacial grimacing and dyskinesia. He had a blank stare, with tongue thrust, frequent daily episodes of extension posturing of the right upper extremity and twisting of the right leg, each lasting for several minutes and causing frequent fall, episodic physical outburst, incoherent speech and insomnia. 
There were bouts of central hypoventilation with tachycardia and daytime incontinence. He remained afebrile and normotensive throughout the hospitalization. His complete blood count showed white blood count (WBC) 4.7, hemoglobin 13.6 g, platelets 153, erythrocyte sedimentation rate $10 \mathrm{~mm} / \mathrm{hr}$; liver, renal, and bone profiles were normal. CSF analysis revealed a lymphocytic pleocytosis with WBC 8, RBC 500 /cumm, as well as negative gram stain and culture. Protein and glucose were normal. CSF also produces negative results for polymerase chain reaction for viral multiplex DNA, including Epstein-Barr, herpes simplex type I, human herpes-6, adenovirus and enterovirus. Given the concern about viral encephalitis, acyclovir was started empirically.

Owing to the stepwise progression of the neuropsychiatric symptoms, a second CSF done 5 days later on showed no cells, and persistently negative gram stain and culture. Protein and glucose remained normal. Oligoclonal band was negative and lactate level was normal. N-methyl-D-aspartate receptor antibody, anti-glial nuclear antibody type 1 , amphiphysin antibody produced negative results in serum and cerebrospinal fluid. Serum anti-voltage-gated potassium channel antibody produced a negative result. Work-ups for autoimmune vasculitis, metabolic disorders, Hashimoto's thyroiditis, and PANDAS were negative; serology tests for mycoplasma pneumonia and common viruses were also negative.

Electroencephalogram (EEG) showed diffuse delta slowing with no epileptiform discharges. Brain MRI and MRA were normal. Due to the stepwise worsening of his symptoms noted by the end of the first week of hospitalization, he received combined immunotherapy for presumed autoimmune encephalitis in the form of pulse methylprednisolone $(25 \mathrm{mg} / \mathrm{kg} /$ day $)$ for three consecutive days, and intravenous immunoglobulin $1 \mathrm{~g} / \mathrm{kg}$ /day for two consecutive days, which resulted in moderate improvement of his symptoms. CT scan of chest, abdomen and pelvis did not reveal any tumors.

His psychiatric symptoms were controlled with risperidone. His movement disorder improved and eventually resolved on carbamazepine. His seizures achieved control on triple medications of phenobarbitone, carbamazepine and topiramate. He was discharged home after 45 days of hospitalization. Follow-up visit at one and 3 months after the discharge revealed a conscious, oriented, and cooperative boy who had a normal mental status with no neurologic deficit. Although he was noted to manifest a drop in ability to concentrate and perform in school, with reading difficulties, sleep disturbance, mood instability, and impulsivity, he continued to show steady improvement with no reported relapses and was described as $80-85 \%$ back to normal by his family at this stage. The phenobarbitone and topiramate were discontinued over the next several months, but he was kept on carbamazepine for one year. A trial of weaning off carbamazepine resulted in recurrence of his seizures. Tumor surveillance and CSF analysis were repeated with a recurrence of seizures and produced a negative result, and he was placed back on carbamazepine. Full recovery was achieved at 9 years of age (3 years after the onset) with above-average performance. There was no reported memory problem, poor concentration, dystonic movement or motor disability.

\section{Discussion}

Anti-NMDA receptor encephalitis is a recently described paraneoplastic syndrome often associated with teratoma or idiopathic autoimmune encephalitis. ${ }^{7,9}$ The paraneoplastic type has been reported in association with ovarian tumors, small cell carcinoma ${ }^{2,3}$ testicular cancer, ${ }^{10}$ or Hodgkin's lymphoma, ${ }^{11}$ while the idiopathic type has been found to be provoked by a viral infection. Herpes simplex virus (HSV) has recently been recognized as a trigger of anti-NMDA encephalitis, and several cases were reported in association with HSV, whereas other reported cases manifested as clinical relapses of HSV owing to anti-NMDA encephalitis. ${ }^{12}$

Most children present with seizures or movement disorders ${ }^{13}$ rather than psychiatric symptoms such as anxiety, agitation, paranoia, and visual or auditory hallucinations, which are more predominant in adults with anti-NMDAAR encephalitis. ${ }^{3,14}$ Due to the presence of refractory partial seizures, the possibility of immune-mediated epilepsies was entertained, including Anti-NMDA receptor encephalitis, limbic encephalitis, refractory seizures associated with GABA receptors antibodies, Rasmussen's encephalitis, and Febrile infection-related epilepsy syndrome (FIRES). The probability for anti-NMDA receptor encephalitis was high owing to the stepwise progression of symptoms, especially the sudden behavioral changes, neurological manifestations and dysautonomia, ${ }^{15}$ consistent with the several cases of anti-NMDA receptors encephalitis that have been reported in the literature (Table 1) ${ }^{3,8}$. In this syndrome, seizures occur in up to $72 \%$ of young children, and focal seizures account for $42 \%$, with a median onset of 15 days before other encephalitis symptoms appear. ${ }^{16}$

Table I The clinical presentation of anti-NMDA receptors encephalitis

\begin{tabular}{|c|c|c|}
\hline Stages & Clinical manifestations & Duration \\
\hline I - Initial & $\begin{array}{l}\text { Viral prodrome } \\
\text { Fever, headache, flu-like symptoms or nausea, vomiting, and diarrhea. }\end{array}$ & Lasts up to $1-2 \mathrm{wks}$ \\
\hline II - Intermediate & $\begin{array}{l}\text { Psychiatric symptoms, i.e. cognitive, memory, behavioral changes, delusions, confusion, } \\
\text { hallucinations, anxiety, emotional disturbances, mania, agitation, sexual disinhibition, } \\
\text { unresponsive/ catatonic state, disorganization, and acute primary psychosis-like schizophrenia. }\end{array}$ & Lasts up to $1-3$ wks \\
\hline III - Prominent & $\begin{array}{l}\text { Neurological symptoms, i.e. movement abnormalities as orofacial - limb dyskinesia's, } \\
\text { dystonic posturing and choreic-like movements of limbs seizures, speech changes, impaired } \\
\text { consciousness, dysautonomia as hyperthermia, tachycardia, hyper-salivation, hypertension, } \\
\text { bradycardia, hypotension urinary incontinence, and central hypoventilation. }\end{array}$ & Lasts from wks to months \\
\hline IV - End & Gradual Recovery & $\begin{array}{l}\text { Expected within months, full } \\
\text { recovery over } 3 \text { or more years }\end{array}$ \\
\hline
\end{tabular}


Typically, an MRI of the brain is either normal or shows nonspecific changes, focal enhancement or medial temporal lobe abnormalities $;{ }^{17}$ EEG often reveals diffuse delta slowing without paroxysmal discharges despite frequent bouts of seizures, ${ }^{17}$ while CSF shows features of inflammation with pleocytosis, increased protein and oligoclonal bands. ${ }^{3}$ The patient in this report had mild CSF and EEG changes consistent with anti-NMDA encephalitis. Since early diagnosis and effective treatment in patients with anti-NMDAR encephalitis have a relatively good prognosis and can achieve baseline recovery and complete resolution at follow-up examination, ${ }^{3,14}$ another CSF analysis was carried out on the $5^{\text {th }}$ day of illness. Serum was taken for auto-antibodies against NMDA, GABA b, AMPA 1 and 2, LG1, and GAD receptors, and he was given combined immunotherapy (Pulse corticosteroid, and IVIG).

Typically, treatment for this syndrome includes first-line immunotherapy with corticosteroids, intravenous immunoglobulin (IVIG), or plasmapheresis, and it is effective in only half of patients. ${ }^{3,5,13}$ Rituximab or cyclophosphamide is usually recommended for the treatment of clinical symptoms if there is no response to the first-line therapy and for prevention of relapse., ${ }^{3,5,13}$ Patient, serum titers were negative as were CSF antibody titers, which is atypical, but given his characteristic neuropsychiatric dysfunction with rapid resolution of symptoms after immunotherapy, he was given a presumptive diagnosis of idiopathic autoimmune encephalitis with presentation like anti-NMDA encephalitis syndrome. A follow-up CSF and serum autoantibodies screen, as well as tumor surveillance, remained negative at 3 and 12 months from the onset.

Shah Rikin et al. ${ }^{18}$ reported two cases with a clinical picture suggestive of anti-NMDA encephalitis and negative antibodies. ${ }^{18}$ One case was a 13.5 years old boy with negative tumor surveillance. The other case was an 8-year-old boy with a benign thymic hyperplasia on surveillance for tumors. Both showed improvement on combined immunotherapy in the form of pulse methylprednisolone therapy and intravenous immunoglobulin, in addition to plasmophoresis in the second case. Their outcome was variable as the first one continued to have psychiatric manifestation and needed monthly immunoglobulin for stabilization, while the other one continued to have motor disability with intermittent dystonia. The patient here responded well with almost complete recovery one year after the event, except for controlled seizures.

The current diagnosis is based upon finding anti-NMDAAR antibodies in the CSF or serum. The CSF antibody has been found to be more sensitive and generally appears to correlate with disease activity. A possible explanation for a false negative result includes smaller quantities of antibodies produced, antigen denaturation during tissue-based immunofixation and variability between human and mouse epitopes used in analysis.

\section{Conclusion}

This case highlights the importance of early recognition of antiNMDA encephalitis-like presentation in patients presenting with seizures, and stepwise progression of neuropsychiatric symptoms, and the importance of prompt immunotherapy treatment even in the absence of antibodies as early therapy improves the long-term neurological outcome of those patients.

\section{Acknowledgments}

This work was supported by the College of Medicine Research Center, Deanship of Scientific Research, King Saud University.

\section{Conflicts of interest}

The author declares no conflict of interest.

\section{References}

1. Granerod J, Tam CC, Crowcroft NS, et al. Challenge of the unknown. A systematic review of acute encephalitis in non-outbreak situations. Neurology. 2010;75(10):924-932.

2. Dalmau J, Tuzun E, Wu HY, et al. Paraneoplastic anti-N-methyl-daspartate receptor encephalitis associated with ovarian teratoma. Ann Neurol. 2007;61(1):2536.

3. Dalmau J, Gleichman AJ, Hughes EG, et al. Anti-NMDA-receptor encephalitis: Case series and analysis of the effect of antibodies. Lancet Neurol. 2008;7(12):1091-1098.

4. Florance NR, Davis RL, Lam C, et al. Anti-N-methyl-d-aspartate receptor (NMDAR) encephalitis in children and adolescents. Ann Neurol. 2009;66(1):11-18.

5. Dalmau J, Lancaster E, Martinez- Hernandez E, et al. Clinical experience and laboratory investigations in patients with anti-NMDAR encephalitis. Lancet Neurol. 2011;10(1):63-74.

6. Kayser MS, Kohler CG, Dalmau J. Psychiatric manifestations of paraneoplastic disorders. AM J Psychiatry. 2010;167(9):1039-1050.

7. Irani SR, Bera $\mathrm{K}$, Waters $\mathrm{P}$, et al. N-methyl-D-aspartate antibody encephalitis: temporal progression of clinical and paraclinical observations in a predominantly non-paraneoplastic disorder of both sexes. Brain. 2010;133(Pt 6):1655-1667.

8. Titulaer MJ, McCracken L, Gabilondo I, et al. Treatment and prognostic factors for long- term outcome in patients with anti-NMDA receptor encephalitis: an observational cohort study. Lancet Neurol. 2013;12(2):157-165.

9. Goldberg EM, Titulaer M, de Blank PM, et al. Anti-N-methyl-Daspartate receptor-mediated encephalitis in infants and toddlers: case report and review of the literature. Pediatr Neurol. 2014;50(2):181-184.

10. Eker A, Saka E, Dalmau J, et al. Testicular teratoma and anti-N-methylD-aspartate receptor-associated encephalitis. J Neurol Neurosurg Psychiatry. 2008;79(9):1082-1083.

11. Zandi MS, Irani SR, Follows G, et al. Limbic encephalitis associated with antibodies to the NMDA receptor in Hodgkin lymphoma. Neurology. 2009;73(23):2039-2040.

12. Armangue T, Titulaer MJ, Málaga I, et al. Pediatric anti-N-methyl-Daspartate receptor encephalitis-clinical analysis and novel findings in a series of 20 patients. J Pediatr. 2013;162(4):850-856.e2.

13. Lin JJ, Lin KL, Hsia SH, et al. Anti-N-methyl-D-aspartate receptor encephalitis in Taiwan - a comparison between children and adults. Pediatr Neurol. 2014;50(6):574-580.

14. Brenton JN, Kim J, Schwartz RH. Approach to the management of pediatric-onset anti- N-Methyl-d-Aspartate (anti-NMDA) receptor encephalitis: a case series. J Child Neurol. 2016;31(9):1150-1155.

15. Lebas A, Husson B, Didelot A, et al. Expanding spectrum of encephalitis with NMDA receptor antibodies in young children. J Child Neurol. 2010;25(6):742-745.

16. Favier M, Joubert B, Picard G, et al. Initial clinical presentation of young children with N-methyl-d-aspartate receptor encephalitis. Eur J Paediatr Neurol. 2018;22(3):404-411.

17. Lizuka T, Sakai F, Ide T, et al. Anti-NMDA receptor encephalitis in Japan: long-term outcome without tumor removal. Neurology. 2008;70(7):504-511.

18. Shah Rikin, Veerapandiyan Aravindhan, Winchester Sara, et al. Two patients with an Anti-N-Methyl-D-Aspartate Receptor Antibody Syndrome-like presentation and negative results of testing for autoantibodies. Pediatric Neurol. 2011;45(6):412-416. 